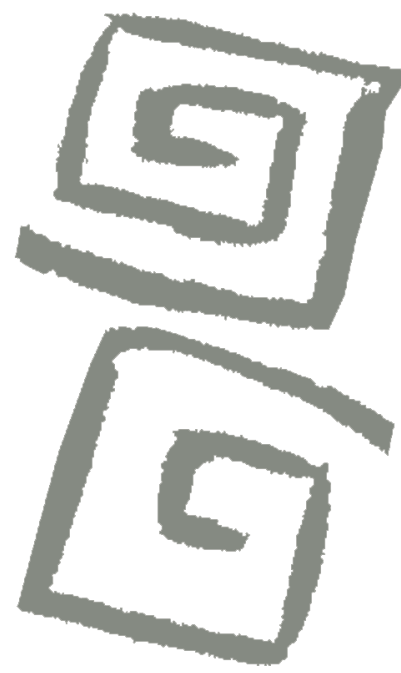

\title{
Prácticas autoreferidas para controlar la hipertensión y la diabetes mellitus en adultos mayores de Campinas, Brasil, en tres períodos
}

\author{
Self-care management practices for arterial hypertension \\ and diabetes mellitus among elderly people in \\ Campinas, Brazil, in three periods
}

Vivian Castro Lemos 1 , Marilisa Berti de Azevedo Barros², Moisés Goldbaum³, Chester Luiz Galvão Cesar', Margareth Guimarães Lima ${ }^{5}$

${ }^{1}$ Autora de correspondencia. Doctoranda en Salud Colectiva, Epidemiología. Departamento de Saúde Coletiva, Faculdade Ciências Médicas, Universidade Estadual de Campinas, Campinas, Brasil. $\square$ (iD

${ }^{2}$ Doctora en Medicina Preventiva. Profesora titular Departamento de Saúde Coletiva, Faculdade Ciências Médicas, Universidade Estadual de Campinas, Campinas, Brasil. $\triangle$ (iD)

${ }^{3}$ Doctor en Medicina Preventiva. Profesor senior Departamento de Medicina Preventiva, Faculdade de Medicina, Universidade de São Paulo, São Paulo, Brasil. $\triangle$ id

${ }^{4}$ Doctor en Salud Pública. Profesor Titular, Departamento de Epidemiologia, Faculdade de Saúde Pública, Universidade de São Paulo, São Paulo, Brasil. $\triangle$ iD

${ }^{5}$ Doctora en Salud Colectiva. Investigadora, Universidade Estadual de Campinas, Campinas, Brasil. $\bowtie$ (iD)
RESUMEN El objetivo fue estimar la prevalencia de las prácticas autoreferidas para controlar la hipertensión y la diabetes, con y sin medicamentos, en adultos mayores de Campinas, Brasil, en tres períodos. Se analizaron los datos de las encuestas de salud realizadas en tres períodos: 2001-2002, 2008-2009 y 2014-2015. La prevalencia de hipertensión, de diabetes, del uso de medicación continua y las prácticas comportamentales aumentaron durante el período analizado, con una caída significativa en el uso no regular de medicamentos y las consultas médicas de rutina en individuos sin plan de salud privado. Los resultados evidenciaron avances en las prácticas relacionadas con la dieta en aquellas personas sin plan de salud y en quienes declararon contar con plan de salud, destacando mejoras en el tratamiento con medicamentos y la práctica de actividad física. La adherencia al uso de medicamentos y a prácticas comportamentales para controlar las morbilidades se mostró consistente en el período evaluado. Estos indicadores refuerzan la necesidad de mantener y ampliar las políticas dirigidas a la educación sanitaria y la asistencia farmacéutica en el país.

PALABRAS CLAVES Cumplimiento y Adherencia al Tratamiento; Adulto Mayor; Hipertensión; Diabetes Mellitus; Brasil.

\begin{abstract}
The objective of this study was to estimate the prevalence of self-care management practices - both with and without medication - in elderly hypertensive and diabetic patients in Campinas, Brazil, in three periods. Data from health surveys conducted in three periods 2001-2002, 2008-2009 and 2014-2015 were analyzed. The prevalence of hypertension, diabetes, the continuous use of medication, and all behavioral practices showed an overall increase in the period analyzed, with a significant drop in both the non-regular use of medications and routine doctor visits on the part of individuals without a private health plan. The results evidenced advances in diet-related practices among individuals without health plans as well as those who reported having healthcare coverage, highlighting improvements in drug treatment and physical activity. Adherence to medication and health behaviors for the management of morbidities was shown to be consistent in the period evaluated. These indicators reinforce the need to maintain and expand policies directed at health education and pharmaceutical assistance in the country. KEY WORDS Treatment Adherence and Compliance; Aged; Hypertension; Diabetes MeIlitus; Brazil.
\end{abstract}




\section{INTRODUCCIÓN}

El envejecimiento poblacional avanza de forma significativa en Brasil, generando debates vinculados a las políticas públicas de salud y a la oferta de servicios, sobre todo respecto de la ocurrencia y control de las enfermedades crónicas no transmisibles ${ }^{(1)}$. Entre estas enfermedades se destacan, por su elevada y creciente frecuencia, la hipertensión arterial y la diabetes mellitus. En 2015, la hipertensión arterial ya alcanzaba al $24,9 \%$ y la diabetes al 7,4\% de las personas de 18 años y más, residentes en las capitales del país ${ }^{(2)}$.

El tratamiento con medicamentos y las acciones no medicamentosas, como la dieta alimentaria y los ejercicios físicos son determinantes para el adecuado control de estas enfermedades $^{(3,4)}$. En lo que respecta al tratamiento con medicamentos, existen diversas opciones de antihipertensivos, antidiabéticos orales y reposición insulínica que propician un manejo adecuado de la presión arterial y de los niveles glucémicos ${ }^{(5,6)}$. En Brasil, la disponibilidad de medicamentos se amplió a partir de la implementación de políticas públicas en la década de 1990, como la Política Nacional de Medicamentos, la Política Nacional de Asistencia Farmacéutica y la Política de Medicamentos Genéricos ${ }^{(7,8)}$. Incluso, entre 2004 y 2006, los programas "Farmacia Popular de Brasil" y "Aquí hay Farmacia Popular" aportaron las perspectivas de reducción del gasto en medicamentos y promoción de un acceso más equitativo ${ }^{(8,9)}$.

Ante este panorama, los datos nacionales de la Encuesta Nacional sobre Acceso, Utilización y Promoción del Uso Racional de Medicamentos muestran que el acceso a los medicamentos para tratar enfermedades crónicas es mayor del $90 \%(94,3 \%)$, con una accesibilidad geográfica equivalente en el Sistema Único de Salud (SUS) y del $72 \%$ en el caso de las farmacias privadas. Vale señalar también que la disponibilidad de los medicamentos en el SUS fue del 45\%, en el Programa Farmacia Popular del $67 \%$, y en los estabelecimientos privados del $88 \%{ }^{(10)}$.
Las prácticas comportamentales que no requieren medicamentos para controlar la hipertensión y la diabetes, tal como señala la bibliografía, son bastante efectivas, implican riesgos mínimos y demandan poca inversión ${ }^{(3,4)}$. Tales medidas demandan cambios en el estilo de vida que deben acompañar el tratamiento del individuo en forma constante ${ }^{(5,6)}$. Ante el reconocimiento de los determinantes comportamentales sobre las condiciones de salud y la calidad de vida, se creó en 2006 la Política Nacional de Promoción de la Salud con el propósito de priorizar la alimentación saludable, practicar actividad física, y disminuir el consumo del tabaco y del alcohol ${ }^{(11)}$.

La Política Nacional de Promoción de la Salud se encuentra en constante revisión e implementación, fomentando la realización de acciones y diversos programas de promoción de la salud, para lo cual el Ministerio de Salud transfirió, en el período 2006-2010, más de 170 millones de reales a los estados y municipios de todo el país ${ }^{(12)}$. Además, un trabajo reciente mostró que más de la mitad de la población brasileña declaró estar vinculada a las Unidades de Salud Familiar, un potente instrumento estratégico de organización de la atención primaria en Brasil ${ }^{(13)}$.

En diversos países y también en Brasil, distintas encuestas poblacionales de salud han monitoreado el uso de servicios por parte de usuarios con o sin planes de salud ${ }^{(14,15,16)}$. Esta utilización está permeada por una multiplicidad de dimensiones, tanto geográficas, como de elecciones personales, disponibilidad de servicios y recursos financieros ${ }^{(17)}$. Sin embargo, hay pocas publicaciones científicas que analicen un conjunto de medidas para controlar estas dos importantes morbilidades, y comparen tres períodos. Hemos hallado solo un estudio con base poblacional llevado a cabo en la ciudad de San Pablo que ha considerado estos aspectos en un único contexto, con poco progreso en el período evaluado ${ }^{(18)}$.

En este sentido, es importante conocer el posible avance en el manejo de la hipertensión y la diabetes, considerando las políticas relacionadas con la promoción de la salud y la asistencia farmacéutica en una metrópolis de un país de altos y medianos ingresos como es 
Brasil. El objetivo de este trabajo fue comparar la prevalencia de prácticas autoreferidas para controlar la hipertensión y la diabetes, con y sin medicamentos, en adultos mayores, entre los períodos 2001-2002, 2008-2009 y 2014-2015 en Campinas, San Pablo, Brasil, y verificar las diferencias en los años 20082009 y 2014-2015 según si cuenta o no con plan privado de salud, lo que permite conocer el perfil de adhesión a las medidas para controlar la hipertensión y la diabetes por parte de la población usuaria de los servicios públicos y privados de salud.

\section{MATERIALES Y MÉTODOS}

En este estudio se analizaron datos de la población de adultos mayores con 60 años y más, resultantes de la Encuesta Multicéntrica de Salud realizada en el estado de San Pablo (ISA-SP, por sus siglas en portugués), en 2001 y 2002, y de la Encuesta de Salud del municipio de Campinas (ISA-Camp, por sus siglas en portugués), correspondiente a los años 20082009 y 2014-2015. En las tres encuestas se recolectaron datos de la población residente en la zona urbana de Campinas, una gran metrópolis brasileña del estado de San Pablo con una población de 1.080.113 habitantes, según el último censo realizado en el país ${ }^{(19)}$.

Las encuestas son de base poblacional, realizadas con muestreo probabilístico, por conglomerados. La ISA-SP 2001-2002 se realizó en cuatro áreas del estado, sin embargo, para este estudio se analizaron solo los datos de Campinas. La información detallada sobre el proceso de muestreo y los métodos utilizados en esa encuesta han sido publicados en un trabajo anterior ${ }^{(20)}$.

En la ISA-Camp 2008-2009, la muestra estuvo compuesta por tres grupos de edad: adolescentes (10 a 19 años), adultos (20 a 59 años) y adultos mayores (60 años y más). Se sortearon muestras de 1.000 personas para cada dominio. Ese número de entrevistas permitió estimar la variación en la frecuencia de los eventos estudiados con una proporción de 0,50 , un error de muestreo de 5 puntos porcentuales e intervalos de confianza del 95\% (IC 95\%), y un efecto de diseño igual a 2. El muestreo se realizó en dos etapas: censal y domiciliario. En los 50 sectores censales sorteados se seleccionaron -también por sorteo- 2.250 domicilios para entrevistar a adolescentes, 700 para entrevistar a adultos y 3.900 para entrevistar a adultos mayores, considerando una pérdida del $20 \%$.

El proceso de muestreo de la ISA-Camp 2014-2015 fue similar al de la ISA-Camp 2008-2009, con algunas singularidades. Se sortearon 70 sectores censales urbanos, 14 por distrito de salud. Si bien el objetivo fue alcanzar muestras de 1.000 adolescentes, 1.400 adultos y 1.000 adultos mayores, al considerar las tasas de no respuesta se sortearon 2.898 domicilios para encontrar adolescentes, 950 para adultos y 3.326 para adultos mayores.

En las tres encuestas, en cada domicilio sorteado, se entrevistaron a todos los habitantes del grupo etario seleccionado para ese domicilio. Las entrevistas se llevaron a cabo directamente con la persona seleccionada por los entrevistadores entrenados, utilizando un cuestionario precodificado. En la última encuesta, en vez de completar el formulario en papel, se utilizaron tablets con un programa especialmente desarrollado para la investigación.

Las variables independentes analizadas son aquellas que corresponden al intervalo de comparación (2002-2009, 2009-2015 y 20022015) y las dependientes son aquellas relacionadas con las prácticas autoreferidas estudiadas para controlar la hipertensión y la diabetes.

La información sobre hipertensión se basó en la pregunta "¿Algún médico $u$ otro profesional de salud ya le mencionó que tiene hipertensión?". Las prácticas para controlar la hipertensión se evaluaron a través de la pregunta "¿qué hace para controlar la hipertensión?" (pudiendo tener más de una respuesta): usa medicación de rutina; usa medicación solo cuando necesita; hace dieta con restricción de sal; hace dieta para perder peso; hace actividad física. La consulta de rutina fue evaluada con la pregunta: "¿Visita al médico/ servicio de salud regularmente a causa de la hipertensión?". 
Para la diabetes se utilizó la pregunta “¿Algún médico u otro profesional de salud ya le mencionó que tiene diabetes?". Las prácticas para controlar la diabetes fueron tomadas de la pregunta: "¿qué hace para controlar la diabetes?" (con posibilidad de más de una respuesta): usa medicación oral de rutina, usa insulina de rutina, hace dieta para perder peso; hace actividad física. Las respuestas "usa insulina de rutina" y "usa medicación oral de rutina" se agruparon en una única variable definida como "usa medicación oral o insulina", para todos los adultos mayores que respondieron "sí" en una o en ambas. La consulta de rutina fue evaluada con la pregunta: "¿Visita al médico/servicio de salud regularmente a causa de la diabetes?".

Para estudiar la distribución de las prácticas autoreferidas para controlar la hipertensión y la diabetes, según si poseía o no plan de salud, se realizó un análisis de estratificación para los dos períodos en los que el cuestionario contemplaba esta pregunta (2009 y 2015).

Todas las variables dependientes estudiadas formaron parte de las tres encuestas, por lo que las preguntas son equivalentes y válidas para ser comparadas. Las bases de datos de las encuestas se sometieron a la evaluación de consistencia y se unificaron en un único archivo. Para unificar las bases, se renombraron y recodificaron todas las variables utilizadas para que fueran idénticas y mantuvieran las mismas categorías de respuestas. Se creó una nueva variable, relativa al origen de la información, con las siguientes categorías 1) ISA-SP 2002, 2) ISA-Camp 2009 y 3) ISA-Camp 2015. Para considerar el diseño muestral en las tres encuestas y, posteriormente realizar el análisis con la debida ponderación, se renombraron las variables referentes al peso final y las variables de unidad primaria de muestreo para que se tornen idénticas y compatibles ${ }^{(21)}$.

Se estimaron las prevalencias e intervalos de confianza del 95\% (IC95\%) para las variables analizadas en cada período y se evaluaron las diferencias estadísticas a través de la prueba de chi cuadrado con ajuste de Rao-Scott ${ }^{(22)}$, para obtener inferencias válidas usando diseños complejos que incluyan estratificación, conglomerados y ponderación como las encuestas de salud, y producir correcciones más robustas en el valor de $p$.

También se estimó la razón de prevalencia (RP) por medio de la regresión de Poisson con intervalos de confianza del 95\% (IC $95 \%$ ), para lo cual se aplicó un modelo de regresión para cada variable del evento analizado. Se ajustó cada modelo por las variables sexo y edad, por considerar que estas variables son potentes determinantes de la salud de los adultos mayores. El análisis se realizó con el software STATA 15.0 que considera el módulo survey del muestreo de las encuestas.

Todos los participantes firmaron los términos del consentimiento libre, previo e informado. El proyecto de la ISA-SP fue aprobado por el Comité de Ética en Investigación de la Facultad de Salud Pública de la Universidad de San Pablo y de la Facultad de Ciencias Médicas de la Universidad Estatal de Campinas (parecer No. 369/2000). A su vez, los proyectos de la ISA-Camp 2008-2009 (parecer No. 079/2007) y de la ISA-Camp 20142015 (parecer No. 409.714/2013) fueron aprobados por el Comité de Ética en Investigación de la Facultad de Ciencias Médicas de la Universidad Estatal de Campinas. El presente estudio fue aprobado bajo el parecer No. 62580716.7.0000.5404.

\section{RESULTADOS}

Entre los adultos mayores sorteados en los domicilios, hubo una pérdida del $9,4 \%$ en $2001-2002,14,2 \%$ en $2008-2009$ y $15,5 \%$ en 2014-2015, incluyendo las personas que se rehusaron a participar y quienes no fueron encontrados en el domicilio luego de varios intentos. Se entrevistaron 426 adultos mayores en 2002, con un mayor porcentaje del sexo femenino $(57,4 \%)$ y una media de edad de 69,8 años [IC 95\% $(68,6-71,0)]$. En 2009, formaron parte de la encuesta 1.519 adultos mayores, en su mayor parte también mujeres $(57,2 \%)$, con una media de edad de 69,9 años [IC 95\% (69,3-70,6)]. En el último estudio realizado en 2015, se entrevistaron 
986 adultos mayores, de los cuales 599 eran mujeres $(57,6 \%)$ y la media de edad fue de 70 años [IC 95\% $(69,2-70,7)]$.

En el análisis ajustado por sexo y edad, la prevalencia de hipertensión autoreferida aumentó un $13 \%$ de 2002 a 2015 y un $10 \%$ de 2009 a 2015. El porcentaje de consultas de rutina para el control de la hipertensión subió un $21 \%$ de 2002 a 2009 , y un $17 \%$ de 2002 a 2015. El uso de medicación regular aumentó un $8 \%$ de 2002 a 2009 y un $10 \%$ entre 2002 y 2015. En el caso del uso de medicación de forma eventual, se observó una caída gradual, principalmente de $2002(9,4 \%)$ a 2009 $(3,1 \%)$ (Tabla 1). Entre los que declararon restringir la sal en la dieta, los resultados mostraron un aumento del $47 \%$ de 2009 a 2015 y al comparar con el período 2002 a 2015, el aumento fue aún más significativo $[R P=1,85$; IC $95 \%(1,29-2,65)]$. Sin embargo, la variable "Hace dieta para perder peso" presentó baja prevalencia, con una disminución de 2002 a 2009. En lo referente a la actividad física, la prevalencia fue del $79 \%$ superior en 2009 respecto de 2015, y dos veces mayor en $2015[R P=2,01$; IC $95 \%(1,11-3,63)]$ con relación a 2002 (Tabla 2).

La prevalencia de diabetes entre las personas encuestadas aumentó significativamente del $15 \%$ al $21,7 \%$ entre 2002 y 2009, alcanzando el 26,2\% en 2015 (Tabla 1). Respecto a las prácticas autoreferidas para controlar la enfermedad se obtuvieron los siguientes resultados: con relación a las consultas de rutina, se observó un aumento del $28 \%$ entre 2002 y 2009 y del $23 \%$ entre 2002 y 2015; el uso de medicación oral o de insulina creció el $15 \%$ de 2009 a 2015 y un $27 \%$ entre los extremos del período; el porcentaje de personas que refirieron hacer dieta para perder peso aumentó más de cuatro veces entre 2009 y 2015 y ocho veces entre 2002 y 2015; la prevalencia de la actividad física aumentó significativamente entre 2002 y 2015 , con una razón de prevalencia de 5,50 [IC 95\% $(1,35-22,3)]$ (Tabla 2).

Al comparar las prevalencias en el período 2009-2015 se verificó que, en aquellas personas sin plan de salud, la prevalencia de las consultas de rutina entre las personas

Tabla 1. Prevalencia de hipertensión y diabetes y prácticas autoreferidas para controlar la hipertensión y la diabetes en personas con 60 años y más, Campinas, San Pablo, Brasil. Años 2002, 2009 y 2015.

\begin{tabular}{|c|c|c|c|c|c|c|c|}
\hline Variables & $\begin{array}{c}2002 \\
(n=426) \\
\%\end{array}$ & IC $95 \%$ & $\begin{array}{c}2009 \\
(n=1.519) \\
\%\end{array}$ & IC $95 \%$ & $\begin{array}{c}2015 \\
(n=986) \\
\%\end{array}$ & $\mathrm{IC} 95 \%$ & Valor de $\mathrm{p}^{*}$ \\
\hline Prevalencia de hipertensión & 51,8 & $46,7-56,8$ & 53,2 & $49,8-56,6$ & 58,7 & $53,1-57,5$ & 0,0272 \\
\hline \multicolumn{8}{|l|}{ Prácticas para controlar la hipertensión } \\
\hline Hace consultas de rutina & 71,5 & $65,2-77,0$ & 86,5 & $83,0-89,4$ & 83,6 & $78,4-87,6$ & 0,0001 \\
\hline Usa medicación de rutina & 86,0 & $80,4-90,2$ & 93,0 & $90,3-94,9$ & 94,4 & $91,8-96,2$ & 0,0004 \\
\hline Usa medicación solo cuando necesita & 9,4 & $5,6-15,3$ & 3,1 & $1,9-5,2$ & 2,2 & $1,3-3,6$ & $<0,0001$ \\
\hline Hace dieta con restricción de sal & 22,0 & $16,0-29,5$ & 27,8 & $22,4-33,8$ & 40,9 & $33,4-48,8$ & 0,0003 \\
\hline Hace dieta para perder peso & 5,3 & $2,8-9,8$ & 2,1 & $1,5-3,1$ & 4,1 & $2,8-6,0$ & 0,0931 \\
\hline Hace actividad física & 7,8 & $4,7-12,7$ & 8,6 & $6,4-11,4$ & 15,5 & $11,3-20,8$ & 0,0056 \\
\hline Prevalencia de diabetes & 15,0 & $11,9-18,6$ & 21,7 & $19,4-24,2$ & 26,2 & $23,2-29,4$ & $<0,0001$ \\
\hline \multicolumn{8}{|l|}{ Práticas para controlar la diabetes } \\
\hline Hace consultas de rutina & 70,7 & $56,5-81,7$ & 90,5 & $86,0-93,7$ & 86,7 & $81,5-90,5$ & 0,0090 \\
\hline Usa medicamento oral o insulina & 72,1 & $61,3-80,4$ & 80,0 & $73,3-85,3$ & 91,8 & $87,9-94,6$ & $<0,0001$ \\
\hline Hace dieta para perder peso & 2,8 & $0,8-8,9$ & 4,6 & $2,3-8,8$ & 22,2 & $15,4-31,1$ & $<0,0001$ \\
\hline Hace actividad física & 2,9 & $0,8-10,8$ & 10,9 & $7,4-15,7$ & 16,0 & $10,6-23,3$ & 0,0101 \\
\hline
\end{tabular}

Fuente: Elaboración propia.

Nota: Número total de personas con 60 años y más entrevistadas en $2002=426$; en 2009=1.519; y en 2015=986.

* Los valores de $p$ fueron estimados por test de chi cuadrado con ajuste de Rao-Scott. 
Tabla 2. Razones de prevalencia e intervalos de confianza de hipertensión, diabetes y prácticas autoreferidas para controlar la hipertensión y la diabetes en personas con 60 años y más. Campinas, San Pablo, Brasil. Años 2002-2009, 2009-2015 y 2002-2015.

\begin{tabular}{|c|c|c|c|c|c|c|}
\hline \multirow{2}{*}{ Variables } & \multicolumn{2}{|c|}{$2002-2009$} & \multicolumn{2}{|c|}{ 2009-2015 } & \multicolumn{2}{|c|}{$2002-2015$} \\
\hline & RP & IC $95 \%$ & RP & IC $95 \%$ & $\mathrm{RP}$ & IC $95 \%$ \\
\hline Prevalencia de hipertensión & 1,02 & $0,91-1,15$ & 1,10 & $1,01-1,20$ & 1,13 & $1,01-1,27$ \\
\hline \multicolumn{7}{|l|}{ Prácticas para controlar la hipertensión } \\
\hline Hace consultas de rutina & 1,21 & $1,10-1,32$ & 0,97 & $0,90-1,03$ & 1,17 & $1,06-1,29$ \\
\hline Usa medicación de rutina & 1,08 & $1,01-1,15$ & 1,02 & $0,98-1,05$ & 1,10 & $1,03-1,16$ \\
\hline Usa medicación solo cuando necesita & 0,34 & $0,17-0,69$ & 0,68 & $0,33-1,42$ & 0,23 & $0,11-0,48$ \\
\hline Hace dieta con restricción de sal & 1,25 & $0,87-1,82$ & 1,47 & $1,11-1,95$ & 1,85 & $1,29-2,65$ \\
\hline Hace dieta para perder peso & 0,40 & $0,19-0,83$ & 0,78 & $0,37-1,65$ & 0,78 & $0,36-1,66$ \\
\hline Hace actividad física & 1,12 & $0,62-2,02$ & 1,79 & $1,17-2,73$ & 2,01 & $1,11-3,63$ \\
\hline Prevalencia de diabetes & 1,45 & $1,13-1,85$ & 1,21 & $1,02-1,42$ & 1,75 & $1,36-2,25$ \\
\hline \multicolumn{7}{|l|}{ Prácticas para controlar la diabetes } \\
\hline Hace consultas de rutina & 1,28 & $1,06-1,54$ & 0,96 & $0,90-1,02$ & 1,23 & $1,02-1,48$ \\
\hline Usa medicamento oral 0 insulina & 1,10 & $0,94-1,29$ & 1,15 & $1,06-1,25$ & 1,27 & $1,10-1,46$ \\
\hline Hace dieta para perder peso & 1,65 & $0,43-6,37$ & 4,85 & $2,25-10,48$ & 8,03 & $2,36-27,25$ \\
\hline Hace actividad física & 3,79 & $0,94-15,24$ & 1,45 & $0,86-2,48$ & 5,50 & $1,35-22,3$ \\
\hline
\end{tabular}

Fuente: Elaboración propia.

Nota: La razón de prevalencia y los intervalos de confianza del 95\% se estimaron por medio de la regresión de Poisson, y se ajustó en todos los casos por sexo y edad.

con hipertensión cayó del 89,8\% en 2009 al $77,2 \%$ en 2015 [RP=0,86; IC $95 \%(0,78-$ $0,95)$ ] y la práctica de dieta con restricción de sal aumentó un $67 \%$. Para las personas con diabetes, además del incremento en la prevalencia de la enfermedad $[\mathrm{RP}=1,40$; IC $95 \%(1,15-1,71)]$, se observó una disminución de la prevalencia de las consultas de rutina, del $91,8 \%$ al $80,4 \%[R P=0,88$; IC $95 \%$ $(0,80-0,96)]$ y un aumento de la prevalencia

Tabla 3. Prevalencia de hipertensión y diabetes y prácticas autoreferidas para controlar la hipertensión y la diabetes en personas con 60 años y más, con 0 sin plan de salud. Campinas, San Pablo, Brasil. Años 2009 y 2015.

\begin{tabular}{|c|c|c|c|c|c|c|c|c|c|c|}
\hline \multirow{3}{*}{ Variables } & \multicolumn{4}{|c|}{ Sin plan } & \multirow[t]{3}{*}{ Valor de $p^{*}$} & \multicolumn{4}{|c|}{ Con plan } & \multirow[t]{3}{*}{ Valor de $p^{*}$} \\
\hline & \multicolumn{2}{|c|}{$2009(n=714)$} & \multicolumn{2}{|c|}{$2015(n=536)$} & & \multicolumn{2}{|c|}{$2009(n=802)$} & \multicolumn{2}{|c|}{$2015(n=449)$} & \\
\hline & $\%$ & IC $95 \%$ & $\%$ & IC $95 \%$ & & $\%$ & IC $95 \%$ & $\%$ & IC $95 \%$ & \\
\hline Prevalencia de hipertensión & 52,5 & $47,4-57,5$ & 57,1 & $52,1-61,9$ & 0,2004 & 53,8 & $50,2-57,4$ & 60,6 & $55,6-65,4$ & 0,0307 \\
\hline \multicolumn{11}{|l|}{ Prácticas para controlar la hipertensión } \\
\hline Hace consultas de rutina & 89,8 & $85,8-92,8$ & 77,2 & $69,7-83,3$ & 0,0005 & 83,4 & $78,2-87,6$ & 90,3 & $85,6-93,5$ & 0,0297 \\
\hline Usa medicación de rutina & 93,4 & $90,6-95,4$ & 92,2 & $87,9-95,0$ & 0,5407 & 92,5 & $88,3-95,3$ & 96,8 & $92,9-98,6$ & 0,0644 \\
\hline Usa medicación solo cuando necesita & 3,0 & $1,7-5,3$ & 3,5 & $1,9-6,3$ & 0,7567 & 3,2 & $1,6-6,3$ & 0,8 & $0,2-2,5$ & 0,0265 \\
\hline Hace dieta con restricción de sal & 26,6 & $20,7-33,4$ & 44,1 & $35,1-53,6$ & 0,0024 & 28,7 & $21,4-37,3$ & 37,4 & $28,3-47,5$ & 0,1690 \\
\hline Hace dieta para perder peso & 2,6 & $1,6-4,2$ & 4,2 & $2,7-6,6$ & 0,1394 & 1,7 & $0,9-3,2$ & 4,0 & $2,4-6,5$ & 0,0332 \\
\hline Hace atividad física & 11,7 & $8,4-16,2$ & 9,3 & $5,9-14,3$ & 0,3999 & 5,8 & $3,7-8,9$ & 22,0 & $15,6-30,2$ & $<0,0001$ \\
\hline Prevalencia de diabetes & 19,8 & $16,7-23,4$ & 27,7 & $24,8-30,8$ & 0,0010 & 23,5 & $20,5-26,9$ & 24,5 & $19,3-30,6$ & 0,7555 \\
\hline \multicolumn{11}{|l|}{ Prácticas para controlar la diabetes } \\
\hline Hace consultas de rutina & 91,8 & $86,0-95,3$ & 80,4 & $73,4-86,0$ & 0,0053 & 89,5 & $82,7-93,8$ & 94,5 & $85,8-98,0$ & 0,2381 \\
\hline Usa medicamento oral o insulina & 81,8 & $72,1-88,6$ & 89,4 & $83,8-93,2$ & 0,0892 & 78,5 & $68,6-86,0$ & 95,0 & $88,9-97,8$ & 0,0007 \\
\hline Hace dieta para perder peso & 4,1 & $1,6-10,1$ & 19,1 & $13,0-27,1$ & 0,007 & 4,9 & $2,1-11,0$ & 26,3 & $15,4-41,2$ & 0,0002 \\
\hline Hace actividad física & 10,3 & $5,6-18,2$ & 8,8 & $4,5-16,4$ & 0,7173 & 11,4 & $7,2-17,6$ & 25,1 & $14,4-40,1$ & 0,0248 \\
\hline
\end{tabular}

Fuente: Elaboración propia. 
Tabla 4. Razones de prevalencia e intervalos de confianza de las prevalencias de hipertensión, diabetes y prácticas autoreferidas para controlar la hipertensión y la diabetes en personas con 60 años y más, con 0 sin plan de salud. Campinas, San Pablo, Brasil. Años 2009 y 2015.

\begin{tabular}{|c|c|c|c|c|}
\hline \multirow[t]{2}{*}{ Variables } & \multicolumn{2}{|c|}{$\underset{2009-2015}{\text { Sin plan }}$} & \multicolumn{2}{|c|}{$\begin{array}{c}\text { Con plan } \\
\text { 2009-2015 }\end{array}$} \\
\hline & $\mathrm{RP}$ & IC $95 \%$ & $\mathrm{RP}$ & IC $95 \%$ \\
\hline Prevalencia de hipertensión & 1,12 & $0,99-1,27$ & 1,10 & $0,99-1,23$ \\
\hline \multicolumn{5}{|l|}{ Prácticas para controlar la hipertensión } \\
\hline Hace consultas de rutina & 0,86 & $0,78-0,95$ & 1,07 & $1,00-1,16$ \\
\hline Usa medicación de rutina & 0,99 & $0,95-1,03$ & 1,04 & $1,00-1,09$ \\
\hline Usa medicación solo cuando necesita & 1,03 & $0,46-2,32$ & 0,23 & $0,60-0,88$ \\
\hline Hace dieta con restricción de sal & 1,67 & $1,21-2,30$ & 1,29 & $0,88-1,89$ \\
\hline Hace dieta para perder peso & 1,63 & $0,86-3,10$ & 2,34 & $1,03-5,33$ \\
\hline Hace actividad física & 0,74 & $0,43-1,28$ & 4,02 & $2,32-6,95$ \\
\hline Prevalencia de diabetes & 1,40 & $1,15-1,71$ & 1,02 & $0,78-1,35$ \\
\hline \multicolumn{5}{|l|}{ Prácticas de control de diabetes } \\
\hline Hace consulta de rutina & 0,88 & $0,80-0,96$ & 1,05 & $0,97-1,15$ \\
\hline Usa medicamento oral o insulina & 1,09 & $0,98-1,23$ & 1,20 & $1,07-1,35$ \\
\hline Hace dieta para perder peso & 4,53 & $1,64-12,47$ & 5,67 & $2,18-14,76$ \\
\hline Hace actividad física & 0,88 & $0,34-2,24$ & 2,40 & $1,25-4,60$ \\
\hline
\end{tabular}

de "hacer dieta para perder peso" del 4,1\% al $19,1 \%$. Entre aquellas personas que contaban con plan privado de salud hubo una disminución del uso irregular de medicamentos $[R P=0,23$; IC $95 \%(0,60-0,88)]$ y un aumento de la prevalencia de la actividad física en los adultos mayores que refirieron tener hipertensión $[\mathrm{RP}=4,02$; IC 95\% $(2,32-6,95)]$. Para las personas con diabetes, hubo un incremento estadísticamente significativo del tratamiento con medicamentos $[R P=1,20, I C$ $95 \%(1,07-1,35)]$, de la frecuencia de hacer dieta para perder peso $[R P=5,67$; IC $95 \%$ $(2,18-14,76)]$, y de la actividad física para el control de la enfermedad $[R P=2,40$; IC $95 \%$ $(1,25-4,60)]$ (Tabla 3 y Tabla 4$).$

\section{DISCUSIÓN}

Se analizaron las prácticas autoreferidas para controlar la hipertensión y la diabetes en adultos mayores de Campinas, San Pablo, Brasil, acompañando su evolución entre los años
2002, 2009 y 2015. Al considerar el período 2002-2015, se verificaron avances significativos en todas las prácticas analizadas para ambas enfermedades. Sin embargo, al evaluar las tendencias entre las personas mayores con cobertura de salud pública y privada, se verificó que, en algunas prácticas, el progreso ocurrió solo en la población con plan de salud.

Las prevalencias de las enfermedades mencionadas presentaron una variación progresiva en el período estudiado (2002-2015), que también fue explicitada por otros autores $^{(18,23,24)}$. Las encuestas Vigitel (Vigilancia de Factores de Riesgo y Protección para Enfermedades Crónicas por Encuesta Telefónica) han monitoreado las frecuencias de enfermedades crónicas, y señalaron estimaciones del $57,7 \%$ de hipertensión y $18,9 \%$ de diabetes, en 2006. Estos indicadores continúan ascendiendo significativamente, sobre todo en adultos mayores, con prevalencias para 2017 del 60,9\% en personas con hipertensión y del $23,5 \%$ en personas con diabetes ${ }^{(25,26)}$.

En este estudio, respecto de las consultas para el seguimiento de la hipertensión y la 
diabetes, las prevalencias aumentaron en el período total evaluado (2002-2015), alcanzando más del $80 \%$ de cobertura en 2015 , para ambas morbilidades, pero con una tendencia de disminución entre 2009 y 2015. Ferreira ${ }^{(27)}$, al evaluar los datos recolectados por la Encuesta Nacional por Muestra de Domicilios 2008, respecto a la no realización de consultas por parte de personas mayores con hipertensión $(10,5 \%)$ y diabetes $(7,1 \%)$, mostró que estas prevalencias estaban por debajo del $11 \%$, lo que reafirma la proximidad con los resultados aquí estimados. Por lo tanto, vale destacar los relevamientos de la Encuesta Nacional por Muestra de Domicilios y de la Encuesta Nacional de Salud que muestran el aumento, a nivel nacional, del acceso y la utilización de los servicios de salud, incluso con variaciones regionales ${ }^{(27,28)}$. En este escenario, es relevante destacar el papel del equipo de atención primaria en el cuidado y monitoreo de las enfermedades crónicas, que da seguimiento a la propuesta del Ministerio de Salud, que incluye actividades de promoción de salud y acompañamiento periódico en las unidades básicas ${ }^{(5,29)}$.

El tratamiento con medicamentos, como dispositivo para controlar las dos morbilidades, se evidenció sobre todo en 2015, con el aumento del $86 \%$ al $94,4 \%$ en las personas con hipertensión y del $72,1 \%$ al $91,8 \%$ en las personas con diabetes, lo que indica una amplia cobertura de medicamentos en esta población. Los resultados de la encuesta telefónica Vigitel, correspondientes al período 2011-2013, también siguieron esta tendencia creciente en adultos mayores con 65 años y más, sobre todo entre las personas con diabetes, pasando del $85,8 \%$ al $93,1 \%{ }^{(30)}$. Los datos de la Encuesta Nacional de Salud indicaron, de forma similar, valores elevados para el tratamiento con medicamentos en la región sudeste entre adultos mayores de 65 a 74 años (90,5\% con hipertensión y $91,9 \%$ con diabetes) ${ }^{(31)}$. En el estudio de Stopa et al. ${ }^{(18)}$, al comparar la razón de prevalencia encontrada en el período 2003-2008, en la encuesta realizada en la ciudad de San Pablo, se encontraron porcentajes de aumento cercanos a los hallados en este trabajo $(R P=1,06$ en personas con hipertensión y $\mathrm{RP}=1,19$ con diabetes); sin embargo, al comparar el período completo evaluado (2003-2015), para las personas con hipertensión, los autores no encontraron un aumento significativo, sino solo en las personas con diabetes, con un incremento del $38 \%$.

En consonancia con el compromiso de garantizar el acceso al tratamiento de las principales enfermedades crónicas, la ampliación del programa Farmacia Popular en 2011, a través de la campaña "La salud no tiene precio", garantizó el acceso gratuito a los antihipertensivos y antidiabéticos ${ }^{(32,33)}$, más allá de la oferta de estos fármacos en la red de las unidades básicas del sistema público de salud brasileño, lo que puede justificar este contexto $^{(34)}$. Se estima que desde el inicio de la campaña, en 2011, hasta el año 2013, aproximadamente el $60 \%$ de los medicamentos para controlar la hipertensión y más del $70 \%$ de los medicamentos para controlar la diabetes fue obtenido en forma gratuita por la población adulta brasileña, tanto en unidades de salud o a través del Programa Farmacia Popular de Brasil ${ }^{(35)}$.

El análisis de los datos provenientes de la Encuesta Nacional sobre Acceso, Utilización y Promoción del Uso Racional de Medicamentos también refuerza la importancia de las políticas nacionales de medicamentos, dado que indica que casi la mitad de las personas mayores encuestadas tienen acceso gratuito a todos los medicamentos prescritos en el tratamiento de enfermedades crónicas, incluso altos porcentajes de acceso a medicamentos para diabetes y enfermedades cardiovasculares $^{(35)}$. El avance de las políticas brasileñas de asistencia farmacéutica reafirman la necesidad de su continuidad y expansión, dado que pueden proporcionar mejores condiciones de salud a los adultos mayores asistidos por el Sistema Único de Salud (SUS) ${ }^{(32,35)}$. La falta de adhesión al tratamiento de enfermedades crónicas puede desencadenar diversas complicaciones, por lo que es fundamental la responsabilidad de los profesionales de la salud para concientizar el autocuidado de los portadores de estas condiciones ${ }^{(36)}$.

Al analizar las prácticas autoreferidas para controlar la hipertensión sin medicamentos, 
se verificó que las prevalencias de la actividad física y de la dieta con restricción de sal prácticamente se duplicaron en los 12 años de estudio. Esto sugiere avances en la comunicación y divulgación -tanto por parte de los profesionales de la salud como por otras fuentes, como la televisión e Internet ${ }^{(37)}$ - de la importancia de sostener prácticas más saludables. Un estudio realizado con los datos de la encuesta nacional de 2013 mostró un porcentaje superior al $90 \%$ de personas con hipertensión que mencionaron haber recibido recomendaciones, en establecimientos públicos y privados, para ingerir menos sal, lo que puede indicar que la comunicación ha mejorado a partir de la implementación de la Política Nacional de Promoción de la Salud, en 2006, y otros programas de promoción de la salud ${ }^{(38)}$. Sin embargo, al comparar los resultados de 2003 y 2009 de la Encuesta de Presupuesto Familiar, respecto al uso de sal en la población brasileña, se encuentran niveles elevados de utilización de acuerdo con las recomendaciones de la Organización Mundial de Salud (OMS) ${ }^{(39)}$. El consumo excesivo de sal puede asociarse a la hipertensión debido a la retención de líquidos que sobrecarga el funcionamiento cardiovascular, aumentando la presión arterial(40).

Respecto de las prácticas autoreferidas para controlar la diabetes, de 2002 a 2015 se encontró un incremento de aproximadamente ocho veces entre quienes refirieron hacer dieta para perder peso y en más de cinco veces entre quienes mencionaron realizar actividad física: mientras en 2002 se encontraron frecuencias de 2,8\% y 2,9\%, en 2015 se estimaron prevalencias del $22,2 \%$ y del $16,0 \%$, respectivamente. Frente a los indicadores alarmantes de sobrepeso en el país ${ }^{(41)}$, y a pesar de que este tipo de abordajes de control sean escasos en las personas mayores, son determinantes en la mejora de la calidad de vida $^{(4)}$.

Diversos autores destacan el efecto hipotensor e hipoglucemiante del ejercicio físico, además de la reducción de la grasa corpo$\mathrm{ral}^{(3,4,42,43)}$. Considerando estos efectos, la construcción de espacios de esparcimiento y para realizar ejercicios físicos, como la creación de los Núcleos de Apoyo a la Salud Familiar y los programas de gimnasios saludables dirigidos a la promoción de la salud, que incluyen la capacitación de los profesionales de la salud, han contribuido a la divulgación y el compromiso de los usuarios ${ }^{(38)}$. Estos espacios deben estar aliñados con la red de atención primaria de la salud, dado que se relacionan con las demandas educativas sobre alimentación y otros cuidados, prácticas de actividades físicas y de esparcimiento, y buscan la participación de la comunidad, especialmente, de grupos en situación de mayor vulnerabilidad social( ${ }^{(44)}$.

Los resultados del presente estudio analizan las prevalencias de las enfermedades y las prácticas de los adultos mayores según si poseen o no plan de salud, lo que permite evaluar si la cobertura asistencial sería equitativa. Desde su creación, el SUS ha mostrado potencialidades de éxito en diversos ámbitos, con una mejor reorientación del proceso salud-enfermedad a través del fortalecimiento de diversas políticas dirigidas al control de las enfermedades crónicas. En este estudio se verificó una disminución de las consultas de los adultos mayores con hipertensión y con diabetes sin cobertura de planes privados de salud, que se condice con otros trabajos que mencionan, además, una mayor prevalencia de las consultas entre los individuos que cuentan con planes de salud lo que se asocia a mayores ingresos y/o escolaridad $(16,27,45,46)$. Aunque la universalización en el ámbito del SUS esté avanzando, las particularidades de los servicios y/o de los sistemas de salud pueden impactar en dificultades individuales de acceso $^{(17)}$.

Al evaluar las demás prácticas autoreferidas para el control de ambas enfermedades estudiadas, en lo que se refiere a los usuarios del SUS o de planes privados de salud, se destaca el aumento de la dieta con restricción de sal en las personas con hipertensión y dieta para perder peso entre las personas con diabetes, sin planes de salud. Por otro lado, en los adultos mayores que mencionaron contar con plan de salud, se encontraron asociaciones con las prácticas relacionadas con el uso de medicamentos, especialmente el uso esporádico, que no se redujo en los individuos 
sin cobertura privada de salud. Este contraste fue similar en los comportamientos relacionados con la actividad física, lo que remite a la necesidad de analizar y reorganizar acciones estratégicas, articulándolas con conductas en el ámbito de la atención primaria del SUS. Otros trabajos también pusieron en evidencia la asociación entre factores de protección de enfermedades crónicas e individuos cubiertos por convenio complementario de salud, relacionados con hábitos alimenticios más saludables y la realización de exámenes preventivos $^{(16,47)}$.

Aunque las políticas implementadas propongan una cobertura universal y la responsabilidad de llegar, sobre todo, a aquellos que no poseen planes de salud, a esta situación se suma la interfaz público-privada, en la que las lagunas que dejan los flujos asistenciales de los seguros privados de salud se superponen al sistema público de salud, y colocan a esta población en desventaja respecto de algunos aspectos de la cobertura ${ }^{(48)}$. En un estudio brasileño, publicado en 2017, se mostraron diferencias significativas al evaluar el uso de los servicios en la esfera del SUS por parte de clientes de planes privados de salud, a los que se les brindaba la oportunidad de acceder a ambos sistemas, tanto al sistema público como al sistema privado de salud ${ }^{(49)}$.

La discusión de los resultados del presente trabajo se torna difícil dada la escasez de estudios con base poblacional que hayan analizado información específica sobre las prácticas para el control de dos morbilidades estudiadas, además de que son raros los trabajos que contemplen el período 2002-2015. Entre las limitaciones, se destaca que toda la información analizada es autoreferida, por lo que está sujeta a sesgos de información, además de la variación en la interpretación de la persona encuestada respecto de la realización de actividad física y dieta. Las encuestas analizadas contemplan números absolutos diferentes, con menor número de información en el período 2001-2002. Esta peculiaridad podría proveer estimaciones menos precisas; sin embargo, fue sensible para detectar diferencias significativas en gran parte del análisis realizado.

Como fortalezas de esta investigación, se destaca que el estudio se llevó a cabo con una muestra representativa de la población de Campinas en tres períodos de comparación, que revelan indicadores importantes sobre el control de las principales morbilidades en el escenario de las enfermedades crónicas no transmisibles. Además, es interesante reforzar la potencialidad de una encuesta de salud a nivel local, que provee información específica y oportuna, que posibilita el monitoreo de los comportamientos y prácticas para controlar importantes enfermedades crónicas, además de poder evaluar el impacto de la implementación de las políticas públicas de salud, que favorezcan la planificación efectiva y la vigilancia de estas enfermedades.

Siendo así, los resultados de la información autoreferida de este trabajo muestran una adhesión continua y creciente en el período evaluado, relacionada al uso de servicios, utilización de medicamentos y comportamientos de salud para controlar tanto la hipertensión como la diabetes. Si bien estos resultados no están estrictamente dirigidos a analizar las políticas de salud, permiten reflexionar sobre la influencia de las políticas de educación para la salud y de medicamentos, que pueden haber incidido positivamente en este contexto, por lo que se recomienda sostener y ampliar tales políticas, que coinciden con la vigilancia, el control y la prevención de las enfermedades crónicas no transmisibles del Sistema Único de Salud de Brasil. 


\section{AGRADECIMIENTOS}

A la Fundação de Amparo à Pesquisa do Estado de São Paulo (FAPESP), por el financiamento del encuesta ISACamp (No. 88/14099, 409747/2006-8, 2012/23324-3); al Ministério da Saúde, Secretaria de Vigilância em Saúde, Secretaria Municipal de Saúde de Campinas, por el apoyo financiero adicional a la encuesta ISACamp (No. 4300, 02-P-28749/2013); a la Coordenação de Aperfeiçoamento em Pesquisa (Capes), por la beca de doctorado de V. C. Lemos; al Conselho Nacional de Desenvolvimento Científico e Tecnológico (CNPq), por la beca de productividad de M. B. A. Barros.

\section{REFERENCIAS BIBLIOGRÁFICAS}

1. Miranda GMD, Mendes ACG, Silva ALA. Population aging in Brazil: current and future social challenges and consequences. Revista Brasileira de Geriatria e Gerontologia. 2016;19(3):507-519. doi: 10.1590/180998232016019.150140 .

2. Brasil. Ministério da Saúde, Secretaria de Vigilância em Saúde, Departamento de Vigilância de Doenças e Agravos não Transmissíveis e Promoção da Saúde. Vigitel Brasil 2015: vigilância de fatores de risco e proteção para doenças crônicas por inquérito telefônico: estimativas sobre frequência e distribuição sociodemográfica de fatores de risco e proteção para doenças crônicas nas capitais dos 26 estados brasileiros e no Distrito Federal em 2015. Brasília: Ministério da Saúde; 2016.

3. Malachias MVB, Souza WKSB, Plavnik FL, Rodrigues $\mathrm{CIS}$, Brandão AA, Neves MFT, et al. $7^{\text {a }}$ Diretriz Brasileira de Hipertensão Arterial. Arquivos Brasileiros de Cardiologia. 2016;107(Supl. 3):S1-S83. doi: 10.5935/ abc. 20160151 .

4. Oliveira JEP, Vencio S, (orgs.). Diretrizes da Sociedade Brasileira de Diabetes: 2015-2016. São Paulo: Editora Clannad; 2016

5. Brasil, Ministério da Saúde, Secretaria de Atenção à Saúde, Departamento de Atenção Básica. Estratégias para o cuidado da pessoa com doença crônica: diabetes mellitus. Brasília, Ministério da Saúde; 2013. (Cadernos de Atenção Básica No. 36).

6. Brasil, Ministério da Saúde, Secretaria de Atenção à Saúde, Departamento de Atenção básica. Estratégias para o cuidado da pessoa com doença crônica: hipertensão arterial sistêmica. Brasília, Ministério da Saúde; 2013. (Cadernos de Atenção Básica No. 37).

7. Brasil, Ministério da Saúde. Política Nacional de Medicamentos [Internet]. 2001 [citado 10 may 2019]. Disponible en: https://tinyurl.com/ybze43oj.

8. Brasil, Ministério da Saúde. Resolução No. 338, de 6 de maio de 2004: Aprova a política de assistência farmacêutica [Internet]. 2004 [citado 10 may 2019]. Disponible en: https://tinyurl.com/y9etlotv.

9. Costa KS, Francisco PMSB, Barros MBA. Conhecimento e utilização do programa farmácia popular do Brasil: estudo de base populacional no município de Campinas-
SP. Epidemiologia e Serviços de Saúde. 2014;23(3):397408. doi: 10.5123/S1679-49742014000300003.

10. Oliveira MA, Luiza VL, Tavares NUL, Mengue SS, Arrais PSD, Farias MR, et al. Access to medicines for chronic diseases in Brazil: a multidimensional approach. Revista de Saúde Pública. 2016;50(Suppl 2):S1-S6. doi: 10.1590/s1518-8787.2016050006161

11. Brasil, Ministério da Saúde, Secretaria de Vigilância em Saúde, Secretaria de Atenção à Saúde. Política Nacional de Promoção da Saúde [Internet]. 3a ed. Brasília: Ministério da Saúde; 2010 [citado 10 may 2019]. Disponible en: https://tinyurl.com/yazasvdf.

12. Malta DC, Morais NOL, Silva MMA, Rocha D, Castro AM, Reis AAC, et al. Política Nacional de Promoção da Saúde (PNPS): capítulos de uma caminhada ainda em construção. Ciência \& Saúde Coletiva. 2016;21(6):16831694. doi: 10.1590/1413-81232015216.07572016.

13. Malta DC, Santos MAS, Stopa SR, Vieira JEB, Melo EA, Reis AAC. A Cobertura da Estratégia de Saúde da Família (ESF) no Brasil, segundo a Pesquisa Nacional de Saúde, 2013. Ciência \& Saúde Coletiva. 2016;21(2):327338. doi: 10.1590/1413-81232015212.23602015.

14. Ahluwalia JB, Bolen J, Garvin B. Health insurance coverage and use of selected preventive services by working age women, BRFSS 2006. Journal of Women's Health (Larchmt) 2007;16(7):935-940. doi: 10.1089/ jwh.2007.CDC8.

15. Nelson KM, Chapko MK, Reiber G, Boylo EJ. The association between health insurance coverage and diabetes care; data from the 2000 behavior risk factor surveillance system. Health Services Research. 2005;40(2):361-372. doi: 10.1111/j.1475-6773.2005.00361.x.

16. Malta, DC, Bernal, RTI, Neto, EV, Curci, KA, Pasinato, MTM, Lisbôa, RM, Cachapuz, RF, Coelho, KSC, Santos, FP, Freitas, MIF. Doenças crônicas não transmissíveis e fatores de risco e proteção em adultos com ou sem plano de saúde. Ciência e Saúde Coletiva. 2019;24(8):31593168. doi: 10.1590/1413-81232018248.30742017.

17. Travassos C, Viacava F, Fernandes C, Maria Almeida C. Desigualdades geográficas e sociais na utilização de serviços de saúde no Brasil. Ciência \& Saúde Coletiva. 2000;5(1):133-149. doi: 10.1590/S141381232000000100012 .

18. Stopa SR, Cesar CLG, Segri NJ, Alves MCGP, Barros MBA, Goldbaum M. Prevalência da hipertensão arterial, do diabetes mellitus e da adesão às medidas comportamentais no Município de São Paulo, Brasil, 2003-2015. Cadernos de Saúde Pública. 2018;34(10):e00198717. doi: 10.1590/0102-311×00198717.

19. Instituto Brasileiro de Geografia e Estatística. Cidades do IBGE: Panorama de Campinas [Internet]. 2019 [citado 12 oct 2018]. Disponible en: https://tinyurl.com/ ybtw8can.

20. Alves MCGP, César CLG, Carandina L, Barros MBA, Goldbaum M. Plano de Amostragem: Saúde e condição de vida em São Paulo. En: Inquérito multicêntrico de 
saúde no Estado de São Paulo (ISA-SP). São Paulo: Faculdade de Saúde Pública, Universidade de São Paulo; 2005. p. 47-62.

21. Francisco PMSB, Barros MBDA, Segri NJ, Alves MCGP, César CLG, Carandina L, et al. Comparação das estimativas de prevalência de indicadores de saúde no Município de Campinas, São Paulo, Brasil, nos anos de 2001/2002 (ISA-SP) e 2008/2009 (ISA-Camp). Cadernos de Saúde Pública. 2013;29(6):1149-1160. doi: 10.1590/ S0102-311X2013000600012.

22. Rao, JNK, Scott, AJ. On simple adjustment to chi square tests with sample survey data. The Annals of Statistics. 1987; 15(1):385-397.

23. Malta DC, Iser BPM, Andrade SSCA, Moura L, Oliveira TP, Bernal RTI. Tendência da prevalência do diabetes melito autorreferido em adultos nas capitais brasileiras, 2006 a 2012. Epidemiologia e Serviços de Saúde. 2014;23(4):753-760. doi: 10.5123/S167949742014000400017.

24. Lobo LAC, Canuto R, Dias-da-Costa JS, Pattussi MP. Tendência temporal da prevalência de hipertensão arterial sistêmica no Brasil. Cadernos de Saúde Pública. 2017;33(6):e35316. doi: 10.1590/0102-311×00035316.

25. Brasil, Ministério da Saúde, Secretaria de Vigilância em Saúde, Secretaria de Gestão Estratégica e Participativa. Vigitel Brasil 2006: vigilância de fatores de risco e proteção para doenças crônicas por inquérito telefônico. Brasília: Ministério da Saúde; 2007. (Série G Estatística e Informação em Saúde).

26. Brasil, Ministério da Saúde, Secretaria de Vigilância em Saúde, Departamento de Vigilância de Doenças e Agravos não Transmissíveis e Promoção da Saúde. Vigitel Brasil 2017: vigilância de fatores de risco e proteção para doenças crônicas por inquérito telefônico: estimativas sobre frequência e distribuição sociodemográfica de fatores de risco e proteção para doenças crônicas nas capitais dos 26 estados brasileiros e no Distrito Federal em 2017. Brasília: Ministério da Saúde; 2019.

27. Ferreira DN, Matos DL, Loyola FAI. Absence of routine medical consultation among hypertensive and/or diabetic elders: an epidemiological study based on the Brazilian National Household Survey 2008. Revista Brasileira de Epidemiologia. 2015;18(3):578-594. doi: 10.1590/19805497201500030005.

28. Stopa SR, Malta DC, Monteiro CN, Szwarcwald CL, Goldbaum M, Cesar CLG. Acesso e uso de serviços de saúde pela população brasileira, Pesquisa Nacional de Saúde 2013. Revista de Saúde Pública. 2017;51(Suppl 1):S1-S10. doi: 10.1590/s1518-8787.2017051000074.

29. Paim J, Travassos C, Almeida C, Bahia L, Macinko J. The Brazilian health system: history, advances, and chaIlenges. The Lancet. 2011;377:1778-1797. doi: 10.1016/ S0140-6736(11)60054-8

30. Brasil, Ministério da Saúde, Secretaria de Vigilância em Saúde, Secretaria de Ciência, Tecnologia e Insumos Estratégicos. Vigitel Brasil: vigilância de fatores de risco e proteção para doenças crônicas por inquérito telefônico: estimativas sobre frequência e distribuição sociodemográfica do uso e fontes de obtenção dos medicamentos para tratamento da hipertensão e diabetes nas capitais dos 26 estados brasileiros e no Distrito Federal, 2011 a 2013. Brasília: Ministério da Saúde; 2015.

31. Tavares NUL, Costa KS, Mengue SS, Vieira MLFP, Malta DC, Silva JJB. Uso de medicamentos para tratamento de doenças crônicas não transmissíveis no Brasil: resultados da Pesquisa Nacional de Saúde, 2013. Epidemiologia e Serviços de Saúde. 2015;24(2):315-323. doi: 10.5123/S1679-49742015000200014.

32. Mengue SS, Tavares NUL, Costa KS, Malta DC, Silva JJB. Fontes de obtenção de medicamentos para tratamento de hipertensão arterial no Brasil: análise da Pesquisa Nacional de Saúde, 2013. Revista Brasileira de Epidemiologia. 2015;18(Suppl 2):192-203. doi: 10.1590/1980-5497201500060017.

33. Costa KS, Tavares NUL, Mengue SS, Pereira MA, Malta DC, Silva JJB. Obtenção de medicamentos para hipertensão e diabetes no Programa Farmácia Popular do Brasil: resultados da Pesquisa Nacional de Saúde, 2013. Epidemiologia e Serviços de Saúde. 2016;25(1):33-44. doi: 10.5123/s1679-49742016000100004.

34. Mengue SS, Bertoldi AD, Ramos LR, Farias MR, Oliveira MA, Tavares NUL, et al. Acesso e uso de medicamentos para hipertensão arterial no Brasil. Revista de Saúde Pública. 2016; 25(1):33-44. doi: 10.1590/s15188787.2016050006154.

35. Tavares NUL, Luiza VL, Oliveira MA, Costa KS, Mengue SS, Arrais PSD, et al. Acesso gratuito a medicamentos para tratamento de doenças crônicas no Brasil. Revista de Saúde Pública. 2016;50(Suppl 2):S1-S13. doi: 10.1590/s1518-8787.2016050006161.

36. Saccomann ICR, Neta JGS, Martins BF. Fatores associados à adesão ao tratamento medicamentoso em hipertensos de uma unidade de saúde da família. Revista da Faculdade de Ciências Médicas de Sorocaba. 2015;17(1):21-26

37. Teixeira JF, Goulart MR, Busnello FM, Pellanda LC. Conhecimento e atitudes sobre alimentos ricos em sódio por pacientes hipertensos. Arquivos Brasileiros de Cardiologia. 2016;106(5):404-410. doi: 10.5935/ abc. 20160049 .

38. Szwarcwald CL, Souza JPRB, Damacena GN, Almeida WS, Malta DC, Stopa SR, et al. Recomendações e práticas dos comportamentos saudáveis entre indivíduos com diagnóstico de hipertensão arterial e diabetes no Brasil: Pesquisa Nacional de Saúde (PNS), 2013. Revista Brasileira de Epidemiologia. 2015;18(Suppl 2):S132S145. doi: 10.1590/1980-5497201500060012.

39. Sarno F, Claro RM, Levy RB, Bandoni DH, Monteiro CA. Estimativa de consumo de sódio pela população brasileira, 2008-2009. Revista de Saúde Pública. 2013;47(3):571-578. doi: 10.1590/S00348910.2013047004418.

40. World Health Organization. Global status report on noncommunicable diseases 2010 [Internet]. Geneva: 
WHO, NUT, NCD; 2011 [citado 10 oct 2018]. Disponible en: https://tinyurl.com/ycb2ssqz.

41. Brasil, Ministério da Saúde, Secretaria de Vigilância em Saúde, Departamento de Vigilância de Doenças e Agravos não Transmissíveis e Promoção da Saúde. Vigitel Brasil 2016: vigilância de fatores de risco e proteção para doenças crônicas por inquérito telefônico: estimativas sobre frequência e distribuição sociodemográfica de fatores de risco e proteção para doenças crônicas nas capitais dos 26 estados brasileiros e no Distrito Federal em 2016. Brasília: Ministério da Saúde; 2017.

42. Hickner AC, Racette SB, Binder EF, Fisher JS, Kohrt WM. Supression of whole body and regional lipolysis by insulin: effects of obesity and exercise. The Journal of Clinical Endocrinology and Metabolism. 1999;84(11):217227. doi: 10.1210/jcem.84.11.6137.

43. Pratley RE, Hagberg JM, Dengel DR, Rogus EM, Muller DC, Goldberg AP. Aerobic exercise training induced reductions in abdominal fat and glucose stimulated insulin responses in mild-aged and older men. Journal of the American Geriatrics Society. 2000;48(9):2022-2033. doi: 10.1111/j.1532-5415.2000.tb04780.x.

44. Carvalho FFB, Nogueira JAD. Práticas corporais e atividades físicas na perspectiva da Promoção da Saúde na Atenção Básica. Ciência \& Saúde Coletiva. 2016;21(6):1829-1838. doi: 10.1590/141381232015216.07482016 .
45. Moreira, JPL, Moraes, JR, Luiz, RR. Utilização de consulta médica e hipertensão arterial sistêmica nas áreas urbanas e rurais do Brasil, segundo dados da PNAD 2008. Ciência \& Saúde Coletiva. 2011;16(9):3781-3793. doi: 10.1590/S1413-81232011001000014.

46. Maclnko J, Lima-Costa MF. Horizontal equity in health care utilization in Brazil, 1998-2008. International Journal for Equity in Health. 2012;11(33). doi: 10.1186/1475-9276-11-33.

47. Malta DC, Bernal RTI, Oliveira M. Tendências dos fatores de risco de doenças crônicas não transmissíveis, segundo a posse de planos de saúde, Brasil, 2008 a 2013. Ciência \& Saúde Coletiva. 2015;20(4):1005-1016. doi: 10.1590/1413-81232015204.14712014.

48. Bahia L, Simmer E, Oliveira DC de. Cobertura de planos privados de saúde e doenças crônicas: notas sobre utilização de procedimentos de alto custo. Ciência \& Saúde Coletiva. 2004;9(4):921-929. doi: 10.1590/ S1413-81232004000400013.

49. Malta DC, Bernal RTI, Lima MG, Araújo SSC de, Silva MMA da, Freitas MIF, et al. Doenças crônicas não transmissíveis e a utilização de serviços de saúde: análise da Pesquisa Nacional de Saúde no Brasil. Revista de Saúde Pública. 2017;51(Suppl 1):S1-S10. doi: 10.1590/s15188787.2017051000090.

FORMA DE CITAR

Lemos VC, Barros MBA, Goldbaum M, Cesar CLG, Lima MG. Prácticas autoreferidas para controlar la hipertensión y la diabetes mellitus en adultos mayores de Campinas, Brasil, en tres períodos. Salud Colectiva. 2020;16:e2407. doi: 10.18294/sc.2020.2407.

Recibido: 14 jul 2019 | Versión final: 2 abr 2020 | Aprobado: 9 abr 2020 | Publicado en línea: 17 may 2020

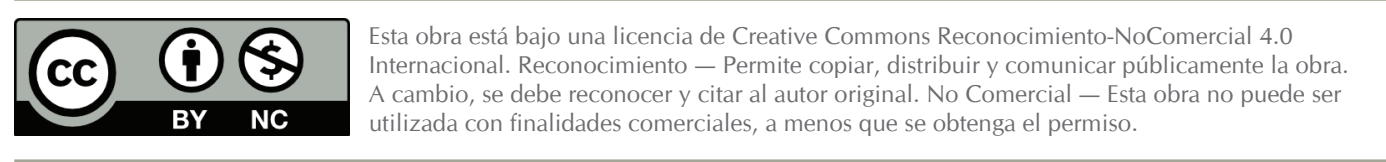

https://doi.org/10.18294/sc.2020.2407 